\title{
Atypical Presentation of Acquired Hypoganglionosis in a Patient Taking Clozapine
}

\author{
Catherine Gisi $\mathbb{D}^{1},{ }^{1}$ Anamaria Munteanu, ${ }^{2}$ Lawrence Ku, ${ }^{1}$ Samuel French, \\ Michael Fleischman, ${ }^{3}$ and Viktor Eysselein ${ }^{3}$ \\ ${ }^{1}$ Department of Internal Medicine, Harbor-UCLA Medical Center, Torrance, CA, USA \\ ${ }^{2}$ Department of Pathology, Harbor-UCLA Medical Center, Torrance, CA, USA \\ ${ }^{3}$ Department of Internal Medicine, Division of Gastroenterology, Harbor-UCLA Medical Center, Torrance, CA, USA
}

Correspondence should be addressed to Catherine Gisi; ckulaga@dhs.lacounty.gov

Received 27 February 2020; Revised 3 June 2020; Accepted 6 June 2020; Published 24 June 2020

Academic Editor: Shiro Kikuchi

Copyright (c) 2020 Catherine Gisi et al. This is an open access article distributed under the Creative Commons Attribution License, which permits unrestricted use, distribution, and reproduction in any medium, provided the original work is properly cited.

Acquired hypoganglionosis (HG) is a rare enteric gastrointestinal neuromuscular disorder previously associated with chronic inflammation that can lead to constipation, ileus, and even death. There is little known about the pathophysiology of acquired hypoganglionosis, and it is unclear if medications are related to the development of the disease. Clozapine is an atypical antipsychotic used to treat refractory schizophrenia that is well known for its side effects including agranulocytosis and gastrointestinal dysmotility. This is an unusual case of acquired hypoganglionosis in a patient with anticholinergic toxicity on clozapine therapy.

\section{Introduction}

Normal gastrointestinal motility depends on appropriate enteric nervous system signaling and coordinated smooth muscle contraction [1]. The enteric nervous system is the largest and most intricate component of the peripheral nervous system. The myenteric (Auerbach's) and submucosal (Meissner's) plexuses contain the majority of neurons and ganglia in the gastrointestinal tract [2]. Acquired hypoganglionosis (HG) is a rare gastrointestinal neuromuscular disease characterized by a decreased number of ganglia in the myenteric and submucosal plexuses [3, 4]. In contrast to congenital hypoganglionosis, such as in Hirschsprung's disease, the acquired form presents in adolescence or in adulthood. Histopathology characteristically demonstrates thickened muscularis mucosa and muscularis propria with decreased numbers of myenteric ganglia [3]. There are multiple theories regarding the pathophysiology of HG. It is primarily thought to be caused by chronic inflammation in the colon [3]. No medications have yet been associated with development of the disease. Differential diagnosis of acquired hypoganglionosis includes chronic intestinal pseudo-obstruction, rare enteric myopathies (mutations of the ACTG2 gene), Hirschprung's disease, Chagas disease, enteric neuropathies, and Waardenburg-Shah syndrome $[5,6]$.

Clozapine is an atypical antipsychotic that is regarded as the most effective treatment for refractory schizophrenia. Despite its efficacy, clozapine remains a third-line medication due to its side effects, including agranulocytosis and severe constipation leading to ileus, gastrointestinal necrosis, and perforation [7]. In January 2020, the FDA strengthened its warning that clozapine-related ileus can lead to serious complications. A review by Cohen et al. reported the casefatality rate for clozapine-related lower gastrointestinal hypomotility to be $15-27.5 \%$, which is about 7 times that of clozapine-related agranulocytosis [8]. Another review reports a mortality rate of $43.7 \%$ due to clozapine-induced ileus from cumulative case reports [9]. Though the mechanism is unknown, it is postulated that clozapine causes constipation due to its anticholinergic, serotonergic, and antihistaminergic properties, similar to other antipsychotics [10].

Presented is a case of acquired hypoganglionosis found in a patient with severe ileus in the setting of the anticholinergic toxicity attributed to clozapine. 


\section{Case Report/Case Presentation}

A 45-year-old Caucasian male with a history of schizoaffective disorder and chronic constipation was brought to the emergency department for acute encephalopathy. He was somnolent and unresponsive to questions. His home medications included a total of $1,200 \mathrm{mg}$ of clozapine daily. It is unclear how long he was taking this dose. He was also taking risperidone, propranolol, linaclotide, benztropine, alprazolam, and diphenhydramine. The patient's medical team could not determine exactly how many years the patient had been taking clozapine, but family and the patient's medical team indicated that he was started on the clozapine therapy years ago in his adulthood. He had never been diagnosed with ileus or other complications of constipation. He had no surgical history. On arrival, the patient was febrile to $38.5^{\circ} \mathrm{Celsius}$ and tachycardic but otherwise hemodynamically stable. Initial abdominal examination was soft, nontender, mildly distended with reduced bowel sounds.

Laboratory studies showed a clozapine level of $735 \mathrm{mcg} /$ $\mathrm{L}$ and norclozapine level of $449 \mathrm{mcg} / \mathrm{L}$ (reference value normal 25-440 mcg/L). Other labs were unremarkable except for a hemoglobin level of $11.8 \mathrm{~g} / \mathrm{dL}$. Cerebrospinal fluid, blood cultures, and urine cultures were negative. Computed tomography (CT) of the head without contrast was unremarkable. He was found to have significant urinary retention on bladder ultrasound. Given the negative infectious workup and his clinical presentation, along with his multitude of medications with anticholinergic side effects, a working diagnosis of anticholinergic toxicity was made, and the patient's home clozapine was held.

On hospital day 2, he developed bilious emesis and physical exam revealed a soft, distended, and tympanic abdomen that was mildly tender. Abdominal CT showed a large stool burden diffusely in the small and large intestines without obvious transition points that was concerning for ileus. The patient underwent aggressive medical therapy including frequent tap water enemas and polyethylene glycol administered via nasogastric tube and per rectum. He remained NPO (nulla per os) and underwent intermittent nasogastric tube suctioning. Large amounts of stool were evacuated, but the patient continued to have bilious vomiting. Repeat abdominal CT showed fecalization of stool in the small intestine and dilatation of the proximal and rectosigmoid colon to $8.4 \mathrm{~cm}$ and $7 \mathrm{~cm}$, respectively, without a clear transition point. The patient was transferred to the intensive care unit and treated with two doses of intravenous neostigmine with significant evacuation of stool. Repeat abdominal CT demonstrated bowel without stool but with significantly worsened dilatation. The patient underwent total colectomy with end ileostomy. Given the remarkable dilatation of the rectum, there was concern that primary ileorectal anastomosis would have led to an inadequate functional outcome, and the decision was made to perform an end ileostomy. The patient recovered rapidly to his mental and functional baseline postoperatively with resumption of normal bowel movements.

Given the patient's severe psychiatric symptoms refractory to other medications, a multidisciplinary meeting with psychiatry was held and it was decided to slowly restart the patient on clozapine with monitoring of symptoms and drug levels. Clozapine was titrated slowly with close monitoring for side effects. The repeat serum clozapine and norclozapine levels were $433 \mathrm{mcg} / \mathrm{L}$ and $121 \mathrm{mcg} / \mathrm{L}$, respectively. There was no recurrence of systemic clozapine toxicity or symptoms of gastrointestinal dysmotility at these lower levels.

Full-thickness sections from the ileum (Figure 1), cecum (Figure 2), appendix, colon, sigmoid, and rectum (Figures 3 and 4) were obtained and stained with hematoxylin and eosin (H\&E), calretinin, and for S-100, a neuronal marker. Final pathologic diagnosis showed hypertrophy of the colonic muscularis propria in the rectum, sigmoid, and cecum. There was focal loss of muscularis propria in the proximal colon. Though there were some ganglia present in the myenteric and submucosal plexuses, they were only focally present in the proximal colon and appendix. Sections from the ileum showed similar muscle hypertrophy and focally absent ganglia in both plexuses consistent with acquired HG.

\section{Pathology}

Full-thickness sections from the ileum, cecum, appendix, colon, and rectum were obtained and stained with hematoxylin and eosin (H\&E) and for S-100, a neuronal marker.

Final pathologic diagnosis showed hypertrophy of the colonic muscularis propria in the rectum, sigmoid, and cecum. There was focal loss of muscularis propria in the proximal colon. Though there were some ganglia present in the myenteric and submucosal plexuses, they were only focally present in the proximal colon and appendix. Sections from the ileum showed similar muscle hypertrophy and focally absent ganglia in both plexuses. Findings were consistent with acquired hypoganglionosis.

\section{Discussion}

There are approximately 100 reported cases of isolated HG distinct from Hirschsprung's disease in the literature in the last 40 years, and only a few of those cases were in Caucasian adults $[3,11]$. It is also associated with considerable morbidity and mortality $[5,11]$. The prevalence of the disease is difficult to quantify because diagnosis is made on histology, which requires examination of full-thickness bowel sections [5]. Immunohistochemistry stains for acetylcholinesterase, S-100, interstitial cells of Cajal, silver, and lactate dehydrogenase are essential in making the diagnosis [11]. It is distinctly different from congenital HG (Hirschsprung's disease), which is diagnosed in children with characteristic contiguous aganglionic segments that start at the anal sphincter and extend proximally [2].

Chronic inflammation in the colon and lower gastrointestinal tract is a common theme unifying theories behind the pathophysiology of acquired HG [3]. Infections, autoimmune diseases, and inflammatory bowel diseases have all been postulated as etiologies of chronic inflammation leading to gut mucosal breakdown and formation of autoantibodies to ganglion cells [3]. Though antipsychotics have 


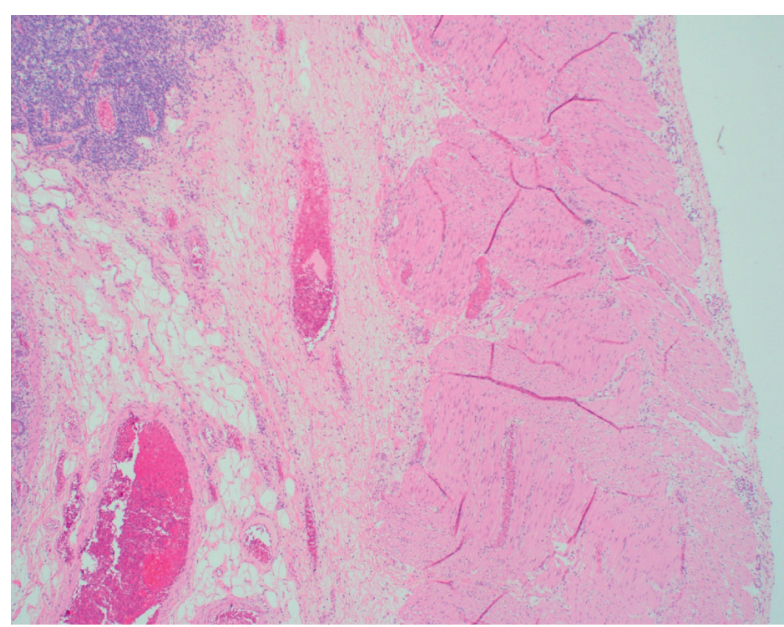

(a)

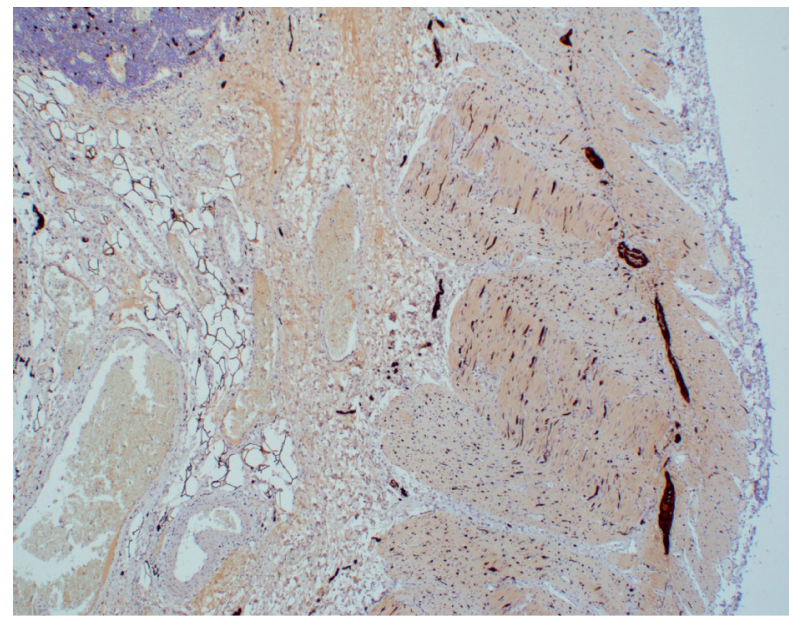

(c)

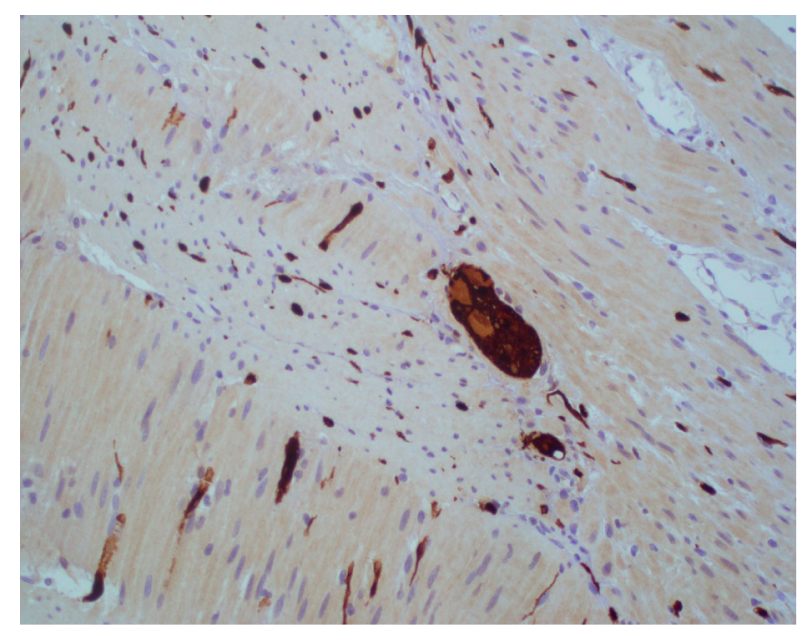

(b)

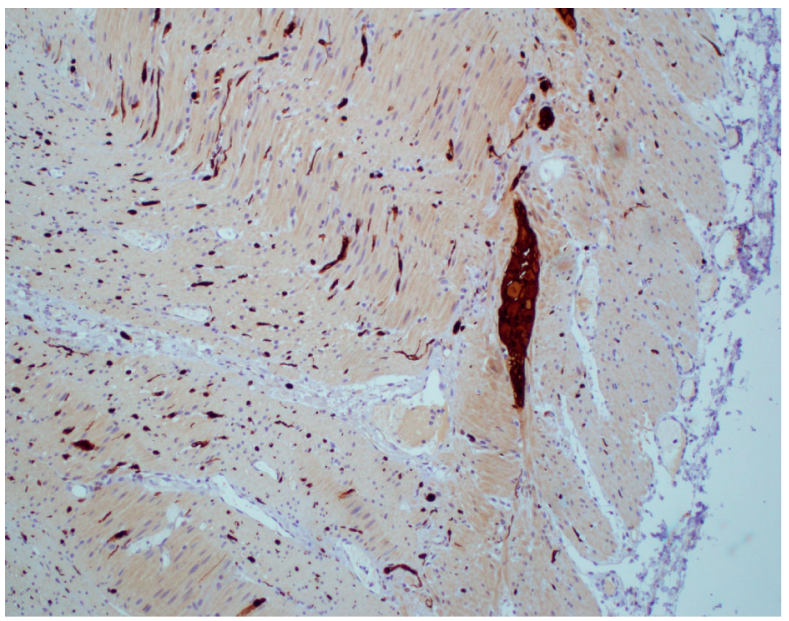

(d)

FiguRE 1: Ileum. (a) Hypertrophy of muscularis propria (magnification 10x, H\&E). (b) Focally absent ganglion cells (magnification 20x, S100). (c) Auerbach's plexus highlighted in dark brown (magnification 10x, S-100). (d) Numerous ganglion cells in some areas (magnification 20x, S100).

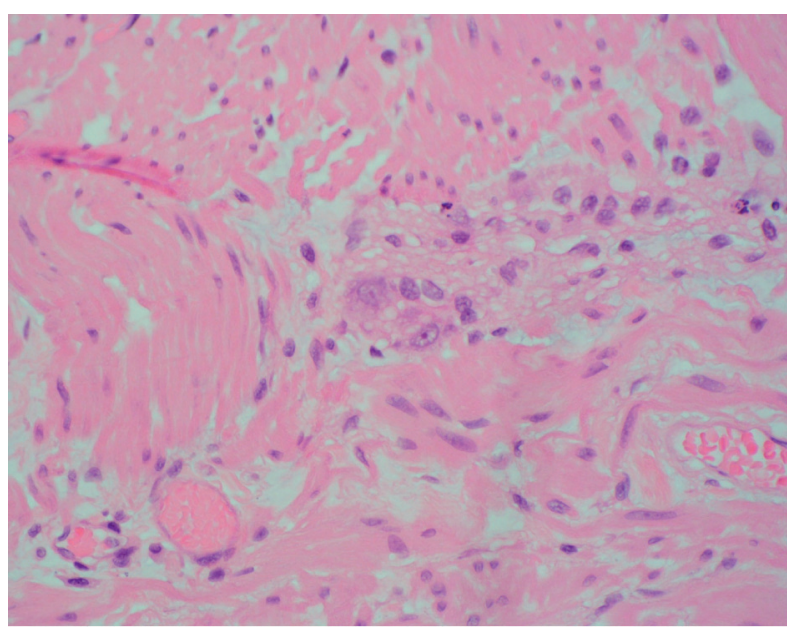

(a)

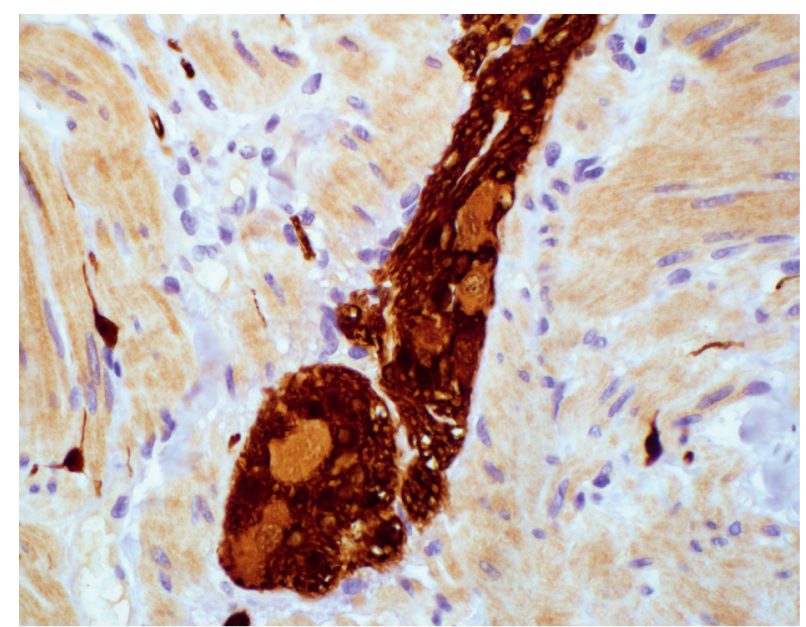

(b)

FIgURE 2: Cecum. (a) Reduced ganglion cells (magnification 40x, H\&E). (b) Reduced ganglion cells (magnification 40x, S100). 


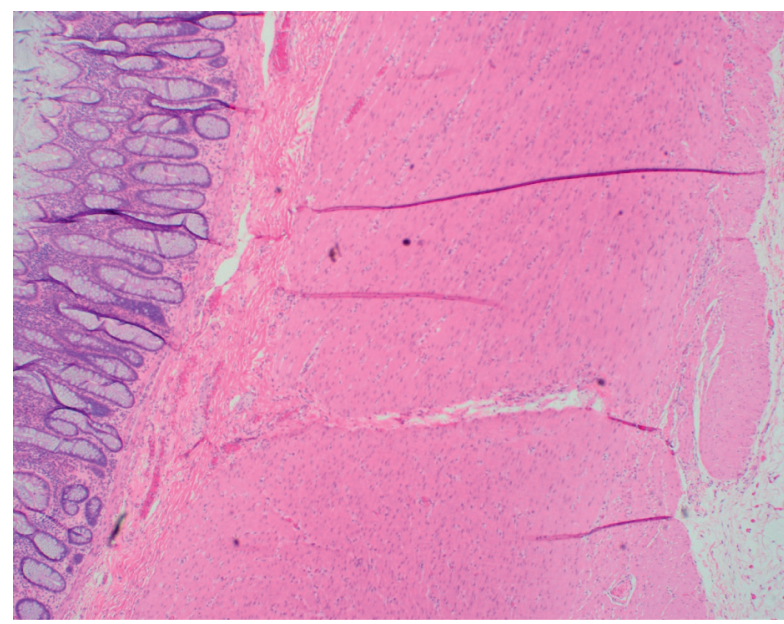

(a)

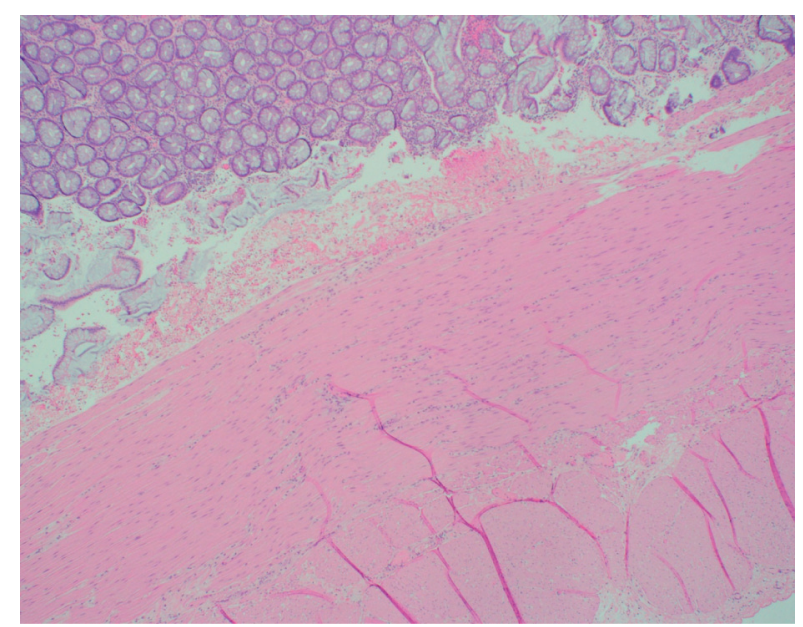

(b)

Figure 3: Hypertrophy of the muscularis propria in rectum and sigmoid sections. (a) Rectum (magnification 10x, H\&E). (b) Sigmoid colon (magnification 10x, H\&E).

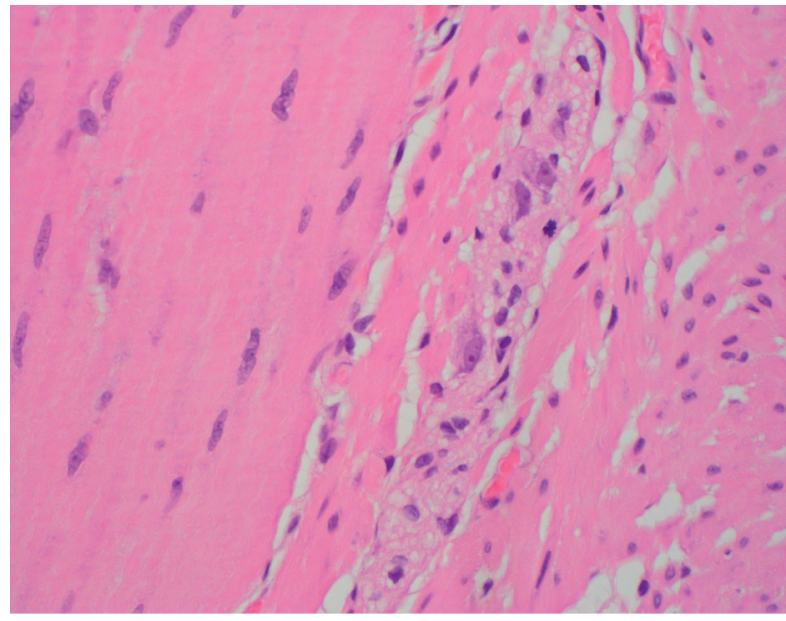

(a)

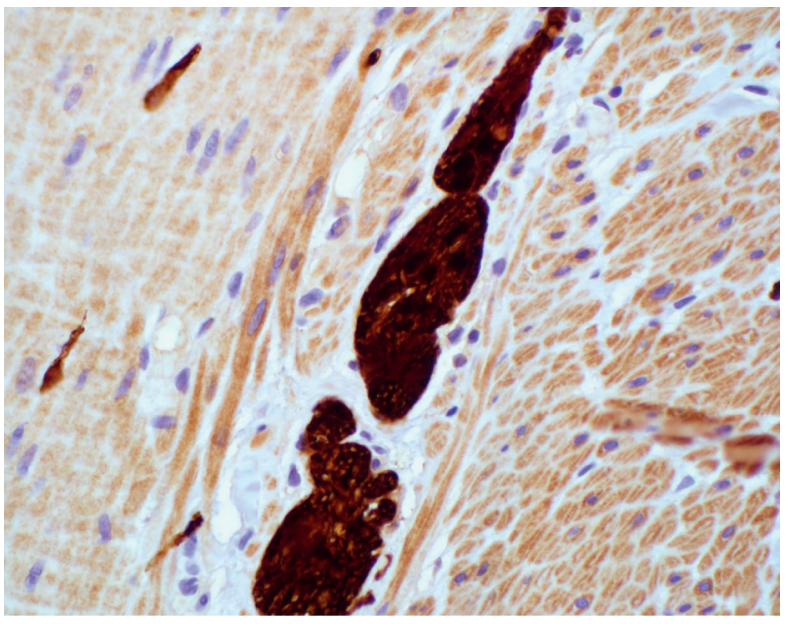

(b)

Figure 4: Sigmoid colon. (a) Focally absent ganglion cells (magnification 40x, H\&E). (b) Focally absent ganglion cells (magnification 40x, S100).

prominent anticholinergic effects and have been known to cause constipation and diarrhea, they have not been previously linked to inflammation [9].

It has been reported that acute infection may dramatically increase the serum concentration of clozapine through downregulation of cytochrome P450 enzymes during inflammation and infection [12]. In this case, no obvious signs of infection were found, but similar cases of severe ileus in the setting of clozapine may warrant close investigation into possible concurrent infections. There are various data on the optimal therapeutic serum levels of clozapine and its more active metabolite, norclozapine $[13,14]$. Serum levels between 350 and $1000 \mathrm{mcg} / \mathrm{L}$ have been associated with greater efficacy and less toxicity, though patients may experience toxicity at various levels, and titration should be individualized with careful monitoring of side effects and symptoms
[14]. In this patient, initial higher levels of clozapine and its metabolite, norclozapine, were associated with severe side effects, which were no longer present when clozapine was stopped. With careful titration and monitoring, the patient achieved lower serum concentrations of clozapine without suffering severe side effects, though this is difficult to interpret because he was postcolectomy.

The Naranjo scale is a validated scoring system for estimating the probability of an adverse drug reaction. In this case, the Naranjo score is 7 , indicating a probable adverse drug reaction [15].

Very few cases of acquired HG have been reported, but the typical presentation of the disease is severe adult-onset ileus with significant colonic distension resulting in colonic necrosis, perforation, and curative colectomy. Pathology in this case did not show classic signs of chronic inflammation 
but was consistent with the typical clinical and histopathologic presentation of acquired hypoganglionosis. It also overlaps with the well-documented presentation of clozapine-related ileus. Despite a large body of literature on clozapine-related ileus, there is a scarcity of data describing the histopathology of this disease [16].

There are numerous reports of fatal clozapine-related ileus in the literature [17]. It is thought that clozapine significantly reduces gastrointestinal motility primarily by affecting the peripheral muscarinic anticholinergic activity via the $\mathrm{M} 3$ receptors in the gut wall [7]. Clozapine is three times more likely to cause constipation than other antipsychotics [5]. One recent study demonstrated that $82 \%$ of patients taking clozapine had dysmotility in at least one region of the GI tract, with more than half of those patients experiencing multiregional dysmotility [18].

In this case, the patient had suffered from constipation for most of his adult life. He had no known history of severe constipation as an adolescent, making congenital aganglionosis unlikely. Given the prevalence of clozapine-induced dysmotility and his severe manifestations of constipation later in adulthood, it is likely that his constipation was caused by years of clozapine and antipsychotic use. The mechanism of clozapine-induced constipation is unknown and difficult to study. One challenge is obtaining tissue for analysis from patients who suffer from clozapinerelated dysmotility and ileus. Colon biopsies are relatively invasive and difficult to obtain. It is possible that many patients taking clozapine have acquired hypoganglionosis but remain undiagnosed because tissue analysis and special stains for ganglionic cells are rarely performed. In this case, this patient would have remained undiagnosed if he had not had catastrophic complications and a subsequent colectomy. More research is needed to determine the pathophysiologic mechanism behind clozapine-induced ileus and the possibility that it is related to acquired hypoganglionosis.

\section{Consent}

The patient and his family have given their written informed consent to publish this case and images.

\section{Conflicts of Interest}

The authors declare that they have no conflicts of interest.

\section{Authors' Contributions}

Catherine Gisi, MD, was involved in conception, data acquisition, interpretation, and drafting of the manuscript.

\section{Acknowledgments}

The authors thank Harbor-UCLA Department of Internal Medicine, Division of Gastroenterology, and Harbor-UCLA Department of Pathology for the support.

\section{References}

[1] K. M. Sanders, S. D. Koh, S. Ro, and S. M. Ward, "Regulation of gastrointestinal motility-insights from smooth muscle biology," Nature Reviews Gastroenterology \& Hepatology, vol. 9, no. 11, pp. 633-645, 2012.

[2] S. Krishnamurthy and M. D. Schuffler, "Pathology of neuromuscular disorders of the small intestine and colon," Gastroenterology, vol. 93, no. 3, pp. 610-639, 1987.

[3] A. M. Kwok, A. B. Still, and K. Hart, "Acquired segmental colonic hypoganglionosis in an adult Caucasian male: a case report," World Journal of Gastrointestinal Surgery, vol. 11, no. 2, pp. 101-111, 2019.

[4] T. Taguchi, K. Masumoto, S. Ieiri, T. Nakatsuji, and J. Akiyoshi, "New classification of hypoganglionosis: congenital and acquired hypoganglionosis," Journal of Pediatric Surgery, vol. 41, no. 12, pp. 2046-2051, 2006.

[5] M. Y. Aldossary, A. Privitera, O. Elzamzami, N. Alturki, and K. Sabr, "A rare case of adult-onset rectosigmoid hypoganglionosis," American Journal of Case Reports, vol. 19, pp. 557-561, 2018.

[6] M. Rao and M. D. Gershon, "Enteric nervous system development: what could possibly go wrong?," Nature Reviews Neuroscience, vol. 19, no. 9, pp. 552-565, 2018.

[7] A. Shirazi, B. Stubbs, L. Gomez et al., "Prevalence and predictors of clozapine-associated constipation: a systematic review and meta-analysis," International Journal of Molecular Sciences, vol. 17, no. 6, p. 863, 2016.

[8] D. Cohen, J. P. A. M. Bogers, D. Van Dijk, B. Bakker, and P. F. J. Schulte, "Beyond white blood cell monitoring," The Journal of Clinical Psychiatry, vol. 73, no. 10, pp. 1307-1312, 2012.

[9] D. Cohen, "Clozapine and gastrointestinal hypomotility," CNS Drugs, vol. 31, no. 12, pp. 1083-1091, 2017.

[10] H. L. Philpott, S. Nandurkar, J. Lubel, and P. R. Gibson, "Drug-induced gastrointestinal disorders," Frontline Gastroenterology, vol. 5, no. 1, pp. 49-57, 2013.

[11] T. C. Deroche, S.-Y. Xiao, and X. Liu, "Histological evaluation in ulcerative colitis," Gastroenterology Report, vol. 2, no. 3, pp. 178-192, 2014.

[12] K. Raaska, V. Raitasuo, M. Arstila, and P. Neuvonen, "Bacterial pneumonia can increase serum concentration of clozapine," European Journal of Clinical Pharmacology, vol. 58, no. 5, pp. 321-322, 2002.

[13] J. C. Ellison and R. L. Dufresne, "A review of the clinical utility of serum clozapine and norclozapine levels," Mental Health Clinician, vol. 5, no. 2, pp. 68-73, 2015.

[14] A. Stark and J. Scott, "A review of the use of clozapine levels to guide treatment and determine cause of death," Australian \& New Zealand Journal of Psychiatry, vol. 46, no. 9, pp. 816-825, 2012.

[15] C. A. Naranjo, U. Busto, E. M. Sellers et al., "A method for estimating the probability of adverse drug reactions," Clinical Pharmacology and Therapeutics, vol. 30, no. 2, pp. 239-245, 1981.

[16] C. A. Janecek, B. C. Morson, S. G. Meuwissen, N. W. Schipper, J. Lindeman, and C. J. Meijer, "Histopathological evaluation of colonic mucosal biopsy specimens in chronic inflammatory bowel disease: diagnostic implications," Gut, vol. 32, no. 12, pp. 1514-1520, 1991.

[17] M. T. Osterman, C. Foley, and I. Matthias, "Clozapine-induced acute gastrointestinal necrosis: a case report," Journal of Medical Case Reports, vol. 11, no. 1, 2017.

[18] S. Every-Palmer, S. J. Inns, E. Grant, and P. M. Ellis, "Effects of clozapine on the gut: cross-sectional study of delayed gastric emptying and small and large intestinal dysmotility," CNS Drugs, vol. 33, no. 1, pp. 81-91, 2019. 